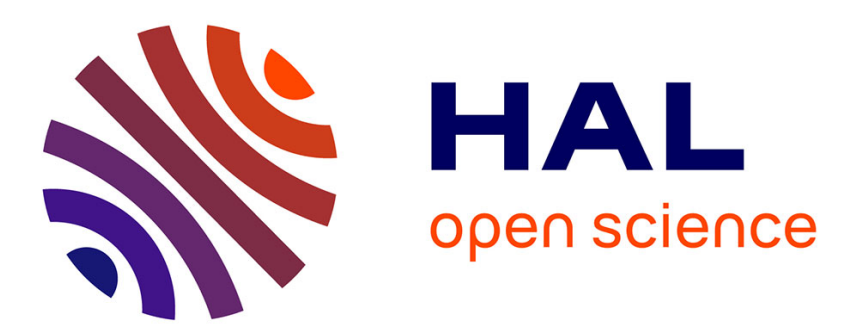

\title{
Fission and Ternary Cluster Decay of Hyper-deformed ${ }^{56} \mathbf{N}$
}

W. von Oertzen, B. Gebauer, G. Efimov, V. Zherebchevsky, S. Thummerer, Tz. Kokalova, Ch. Schultz, H.G. Bohlen, D. Kamanin, C. Beck, et al.

\section{To cite this version:}

W. von Oertzen, B. Gebauer, G. Efimov, V. Zherebchevsky, S. Thummerer, et al.. Fission and Ternary Cluster Decay of Hyper-deformed ${ }^{56}$ N. European Physical Journal A, 2008, 36, pp.279-288. 10.1140/epja/i2008-10592-7 . in2p3-00274397

\section{HAL Id: in2p3-00274397 https://hal.in2p3.fr/in2p3-00274397}

Submitted on 18 Apr 2008

HAL is a multi-disciplinary open access archive for the deposit and dissemination of scientific research documents, whether they are published or not. The documents may come from teaching and research institutions in France or abroad, or from public or private research centers.
L'archive ouverte pluridisciplinaire HAL, est destinée au dépôt et à la diffusion de documents scientifiques de niveau recherche, publiés ou non, émanant des établissements d'enseignement et de recherche français ou étrangers, des laboratoires publics ou privés. 


\title{
Fission and Ternary Cluster Decay of Hyper-deformed ${ }^{56} \mathrm{Ni}$.
}

\author{
W. von Oertzen ${ }^{1}$, B. Gebauer ${ }^{1}$, G. Efimov ${ }^{1,2}$, V. Zherebchevsky ${ }^{1,6}$, S. Thummerer ${ }^{1}$, Tz. Kokalova $^{1}$, Ch. Schulz $^{1}$, H. $^{2}$ \\ G. Bohlen ${ }^{1}$, D. Kamanin ${ }^{1,2}$, C. Beck ${ }^{3}$, D. Curien ${ }^{3}$, P. Papka ${ }^{3}$, M. Rousseau ${ }^{3}$, G. Royer ${ }^{4}$, G. de Angelis ${ }^{5}$ \\ ${ }^{1}$ Hahn-Meitner-Institut-GmbH, Berlin, Germany \\ ${ }^{2}$ Flerov laboratory for Nuclear Reactions, JNR, Dubna, Russia \\ ${ }^{3}$ IPHC, Département de Recherches Subatomiques, IN2P3-CNRS UMR7178 Université Louis Pasteur, Strasbourg, France \\ ${ }^{4}$ Subatech,UMR:Université-IN2P3/CNRS-Ecole des Mines, Nantes, France \\ ${ }^{5}$ Laboratori Nationali di Legnaro, Legnaro, Italy \\ ${ }^{6}$ St. Petersburg University, St. Petersburg, Russia
}

version: $8 / 04 / 2008$

\begin{abstract}
Coincidences between two heavy fragments have been measured from fission of ${ }^{56} \mathrm{Ni}$ compound nuclei, formed in the ${ }^{32} \mathrm{~S}+{ }^{24} \mathrm{Mg}$ reaction at $\mathrm{E}_{l a b}\left({ }^{32} \mathrm{~S}\right)=165.4 \mathrm{MeV}$. A unique experimental set-up consisting of two large area position sensitive $(\mathrm{x}, \mathrm{y})$ gas-detector telescopes has been used allowing the complete determination of the observed fragments, and their momentum vectors. In addition to binary fission events with subsequent particle evaporation, narrow out-of-plane correlations are observed for two fragments emitted in purely binary events and in events with a missing charge consisting of $2 \alpha$ and $3 \alpha-$ particles $\left({ }^{12} \mathrm{C}\right)$. These events are interpreted as ternary cluster decay from ${ }^{56} \mathrm{Ni}$-nuclei at high angular momenta through hyper-deformed shapes.
\end{abstract}

PACS. 2 5.70.Jj, 25.70.Pq, 24.60.Dr

\section{Introduction}

Clustering and large deformations are observed as general phenomena at low excitation energy in light $\mathrm{N}=\mathrm{Z}$ nuclei. At higher excitation energies and higher angular momenta super- and hyper-deformed shapes are predicted (and partially observed) in nuclei with masses ranging from $\mathrm{A}=$ 20 up to 100 , see [1-7]. For these nuclear configurations shell corrections for quadrupole deformation parameters $\beta_{2}=0.6-1.0$, corresponding to major-to-minor axis ratios of 2:1 up to $3: 1$ (for ellipsoidal shapes), play an important role. These states are typically found at energies, which are several $\mathrm{MeV}$ lower than the liquid drop values. At lower angular momentum, the deformed shell corrections will also stabilize the rotating nucleus in its super- and hyper-deformed shapes [5] and in some cases enhanced $\gamma$-transitions can be expected to be observed over several spin values. In the present work the cluster decay at higher angular momentum is considered.

Configurations showing the cluster structure of the hyper-deformed states in ${ }^{56} \mathrm{Ni}$ were obtained with the BrinkBloch $\alpha$-cluster model [2], highlighting the relation between large deformations and clustering. This work suggests that hyper-deformed states in ${ }^{56} \mathrm{Ni}$ will fission into several clusters, in particular at high angular momentum. Furthermore, ternary fission is predicted for such nuclei using an approach based on the generalised liquid drop model [8,9], taking into account the proximity energy and quasi-molecular shapes as obtained in the $\alpha$-cluster model [2]. The ternary cluster decay has, in the present mass range, a less favourable Q-value as compared to the binary mass split, particular binary and ternary mass splits are predicted to be favoured [2] also from $\mathrm{SU}(3)$ cluster-structure considerations [10]. As will be shown in the following, however, ternary cluster decay can be strongly enhanced for the largest deformations due to the lowering of the fission barrier obtained with the large moments of inertia and by the aforementioned shell corrections. Until now experimental evidence for such ternary break-up has only been reported [11-13] by our group for ${ }^{60} \mathrm{Zn}$ with a similar experimental set-up. Searches for hyper-deformation in heavier nuclei via $\gamma$-decay at high angular momentum [15] have shown that its identification is a very difficult task, because the probability of fission will be strongly increased.

We have studied fission events from the decay of the ${ }^{56} \mathrm{Ni}$ compound nucleus $(\mathrm{CN})$ at an excitation energy of $\mathrm{E}_{C N}^{*}=83.8 \mathrm{MeV}$, formed in the ${ }^{32} \mathrm{~S}+{ }^{24} \mathrm{Mg}$ reaction at $\mathrm{E}_{l a b}=163.5 \mathrm{MeV}$. The incident energy was chosen to correspond to the $\mathrm{E}_{C N}^{*}$ of the last broad resonance [16] observed in the ${ }^{28} \mathrm{Si}+{ }^{28} \mathrm{Si}$ channel. In the experiment two heavy fragments are measured in coincidence. The compound nucleus decay in the ${ }^{32} \mathrm{~S}+{ }^{24} \mathrm{Mg}$ system has been studied extensively by Sanders et al. [17]. From this pio- 
neering work some basic information on the CN formation is available. For instance, the maximum angular momentum reached for ${ }^{56} \mathrm{Ni}$, is close to $45 \hbar$, consistent with the predicted liquid-drop limit [14]. At these high angular momenta the binary fission decay can reach up to $10 \%$ of the total fusion cross section, which leads dominantly to evaporation residues. In the present work ternary fission is identified by the coincident registration of two fragments with a measured total charge much lower than the compound nucleus. This is done with a unique experimental set up, which allows the full coverage of the in-plane and out-of-plane angular correlations. The ternary cluster decay in the present case competes at high angular momentum with the binary fission and the evaporation of $3 \alpha$-particles (which have very negative $\mathrm{Q}$-values) and high fission barriers, due to the formation of elongated hyperdeformed configurations and the corresponding lowering of the ternary fission barriers.

\section{The Experiments}

\subsection{Experimental conditions}

The experiment was performed at the VIVITRON Tandem facility of IReS (Strasbourg) with the BRS-EUROBALL set-up [18-20] aimed at particle- $\gamma$-spectroscopy (see Fig. 1). We report here on the results of charged particle spectroscopy, first results on the $\gamma$-spectroscopy will be reported in ref. [21].

Two detector telescopes (see Fig. 2), labelled 3 and 4 , are located symmetrically on either side of the beam axis. Each of them comprises two-dimensional positionsensitive a low-pressure multi-wire chamber (MWC) and a Bragg-curve ionisation chamber (BIC). All detection planes are electrically fourfold segmented, in order to improve the resolutions and counting rates. A scheme is shown in Fig.2, there the arrangement of the position sensitive part and the Bragg-ionisation chambers are shown.

In the reaction, schematically written as: $\left(M_{1}, Z_{1}\right)+\left(M_{2}, Z_{2}\right) \rightarrow{ }^{56} \mathrm{Ni}^{*} \rightarrow\left(M_{3}, Z_{3}\right)+(\Delta \mathrm{Z})+\left(M_{4}, Z_{4}\right)$, two heavy fragments with masses $\left(M_{3}, M_{4}\right)$ and charges $\left(Z_{3}, Z_{4}\right)$ are registered in kinematical coincidence and identified by their charges. Further details of the detectors and the experimental set-up are given in $[21,13]$. The ${ }^{24} \mathrm{Mg}$ targets consisted of $240 \mu \mathrm{g} / \mathrm{cm}^{2}$ enriched to $99.9 \%$ on a layer of $20 \mu \mathrm{g} / \mathrm{cm}^{2}$ of ${ }^{12} \mathrm{C}$. The vacuum in the present case was rather poor, therefore oxidation of the target was appreciable. For the later discussion the potential contributions from oxygen in the target and the ${ }^{12} \mathrm{C}$-backing are of importance.

The two detectors cover in-plane angles, of $\theta_{L}=12.5^{\circ}-$ $45.5^{\circ}$, and in their centre planes the out-of-plane angles $\phi_{L}$ ranges are $\Delta \phi=0^{\circ} \pm 16.8^{\circ}$, or $180^{\circ} \pm 16.8^{\circ}$. The centre-of-mass angular ranges are with the "inverse" kinematics in the region $\theta_{c m}=70^{\circ}-125^{\circ}$. With the position sensitive detectors the correlations have been measured between two heavy ejectiles for in-plane and out-of-plane

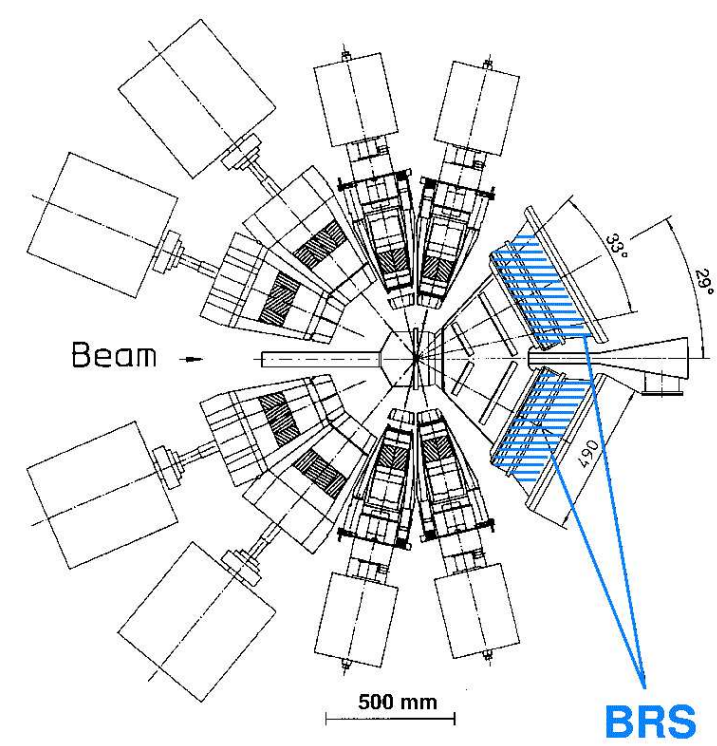

Fig. 1. The BRS-Euroball-IV set-up used for the study of coincident fission channels in the reactions ${ }^{32} \mathrm{~S}+{ }^{24} \mathrm{Mg} \rightarrow Z_{3}+$ $Z_{4}+\Delta Z$, at $\mathrm{E}_{l a b}=163.5 \mathrm{MeV}$.

scattering angles, $\theta$ and $\phi$, respectively over a wide angular range. Other parameters measured are the Bragg-peak height $B P$, the range $R$ and the rest energy $E$, giving the identification of the fragments by their charge and momentum vectors. With the BRS it is therefore possible to measure two heavy fragments in coincidence with respect to their in-plane $\left(\theta_{3}, \theta_{4}\right)$, and out-of-plane $\left(\varphi_{3}, \varphi_{4}\right)$ scattering angles, their time of flight (TOF) and energy (E). In comparison to previous work these coincidences represent an exclusive measurement of the fission yield. A typical two-dimensional spectrum of $B P$ versus $E$ is shown in Fig. 3. An example of a gate in one of the detectors is shown in Fig. 3, from these the inclusive yields are obtained by alternatively setting gates in the detectors 3 or 4 . These yields are shown in Fig. 4 . They have not been corrected for contributions from target contaminants (see below). For large charge asymmetry the efficiency gets below $100 \%$ (see also ref. [12,13]. The absolute values are typically $50 \%$ smaller than those in the previously studied case [13] of ${ }^{36} \mathrm{Ar}+{ }^{24} \mathrm{Mg}$ system. In this case the excitation energy in ${ }^{60} \mathrm{Zn}$ was higher, $88 \mathrm{MeV}$ as compared to 83.8 $\mathrm{MeV}$ in ${ }^{56} \mathrm{Ni}$ in the present case, and the limiting angular momentum is smaller by $3-5 \hbar$

The relation $\left(\phi_{3}-\phi_{4}\right)=180^{\circ}$ for the out-of-plane angles defines coplanarity. The reaction plane is defined by the beam axis and the two vectors of the emitted heavy fragments.

Reaction channels are determined by the the sum of the observed charges of the fragments, $\left(Z_{C N}=Z_{3}+Z_{4}+\right.$ $\Delta Z$ ), with different values of $\Delta \mathrm{Z}=0-8$. This procedure gives information for channels with a well defined missing charge $\Delta \mathrm{Z}=\left(Z_{C N}-Z_{3}-Z_{4}\right)$. For binary exitchannels, with two heavy fragments which do not evaporate particles, narrow out-of-plane $\phi$-distributions must 


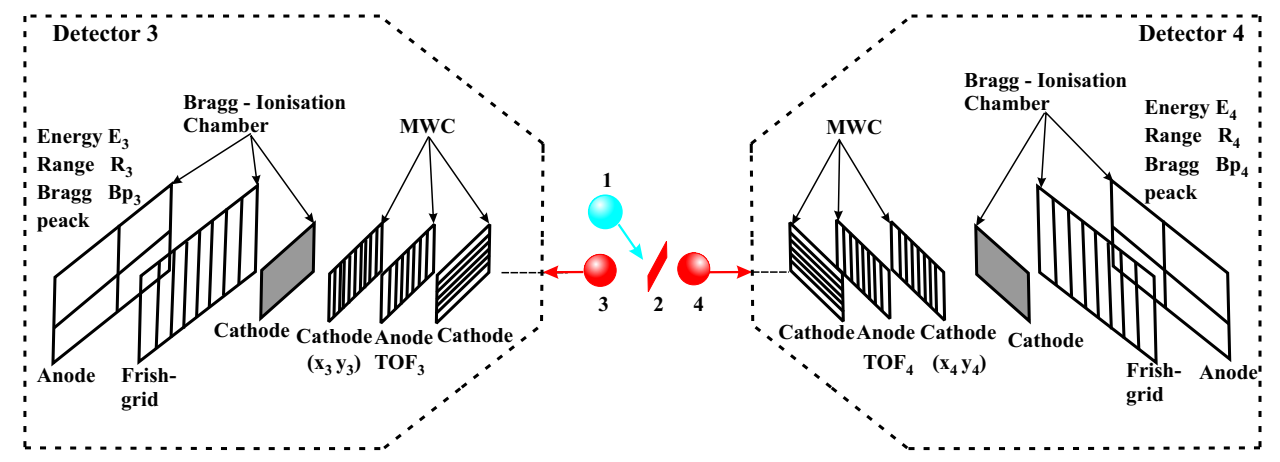

Fig. 2. Details of the position sensitive detector telescopes of the BRS. The position sensitive (X,Y) low-pressure multi-wire (MWC) part and the Bragg-Ionisation Chamber (BIC) part, are indicated.

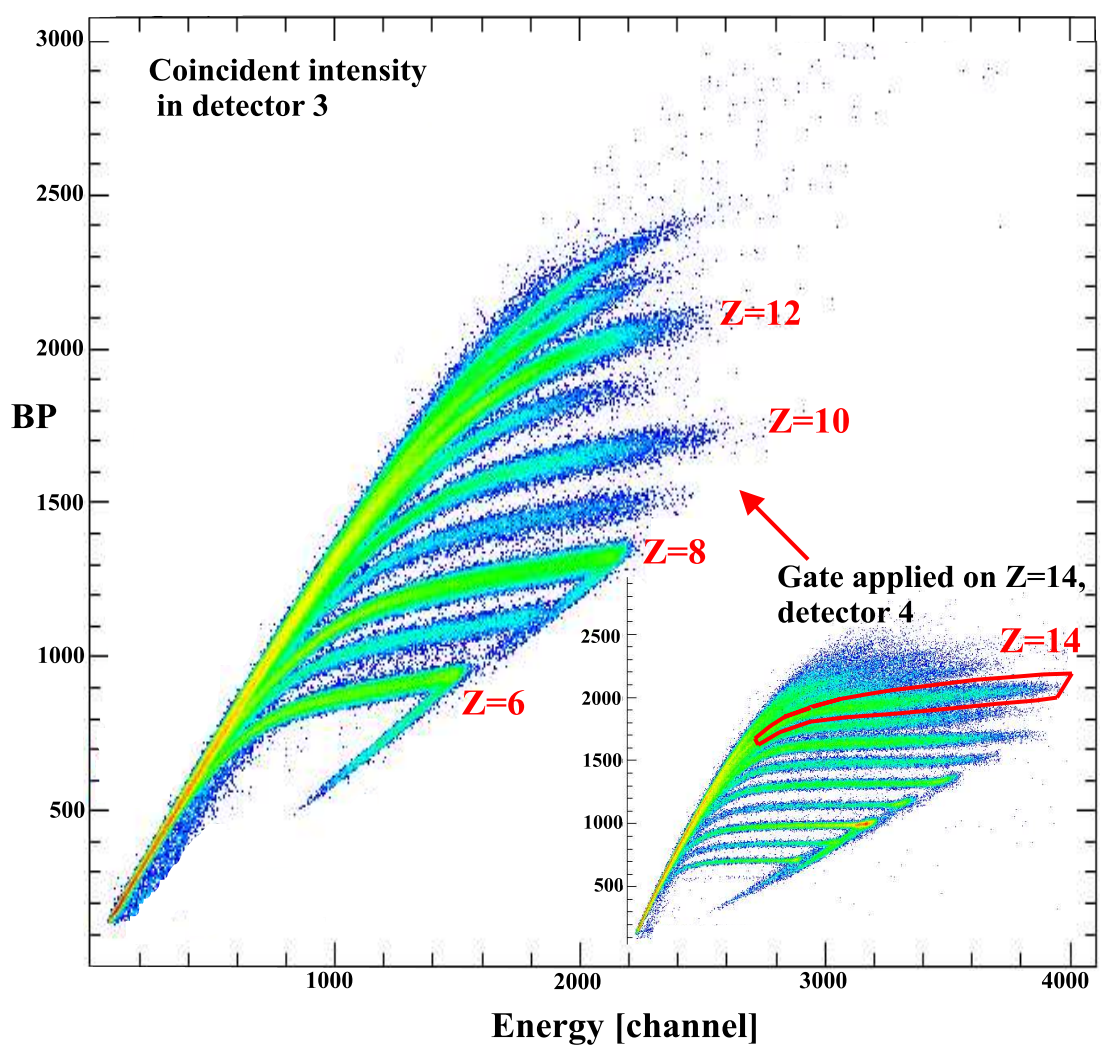

Fig. 3. (Color online) Bragg-peak-Energy distributions of events in detector 3 obtained in coincidence with a $Z=14$ gate in detector 4 as shown in the insert for the reaction ${ }^{32} \mathrm{~S}+{ }^{24} \mathrm{Mg} \rightarrow Z_{3}+Z_{4}+\Delta Z$.

be observed. For non-binary channels with the evaporation of light particles broad distributions (broad in the $\phi$-distributions) appear, because of the missing information on the momenta of the unobserved third particles.

\subsection{Assessment of contributions from contaminants in the target}

For the following discussion of ternary events these outof-plane correlations are essential. The fragment yields,
$N\left(Z_{3}, Z_{4}\right)$, are plotted in Fig. 5 as a function of $\left(\phi_{3}-\right.$ $\left.\phi_{4}\right)$ for some combinations of $Z_{3}$ and $Z_{4}$, with even total charge, but different $\Delta \mathrm{Z}$. The coplanarity condition is fulfilled for purely binary events $\left(Z_{3}+Z_{4}=28\right)$ in the form of a narrow peak around $\left(\phi_{3}-\phi_{4}\right)=180^{\circ}$. The small broader component in the out-of-plane angular correlations is the result of neutron evaporation. No strong narrow correlation is observed for $\Delta \mathrm{Z}=2$ (first column in Fig. 5), where the corresponding recoil of the evaporated $\alpha$-particles widens the angular correlation. These 

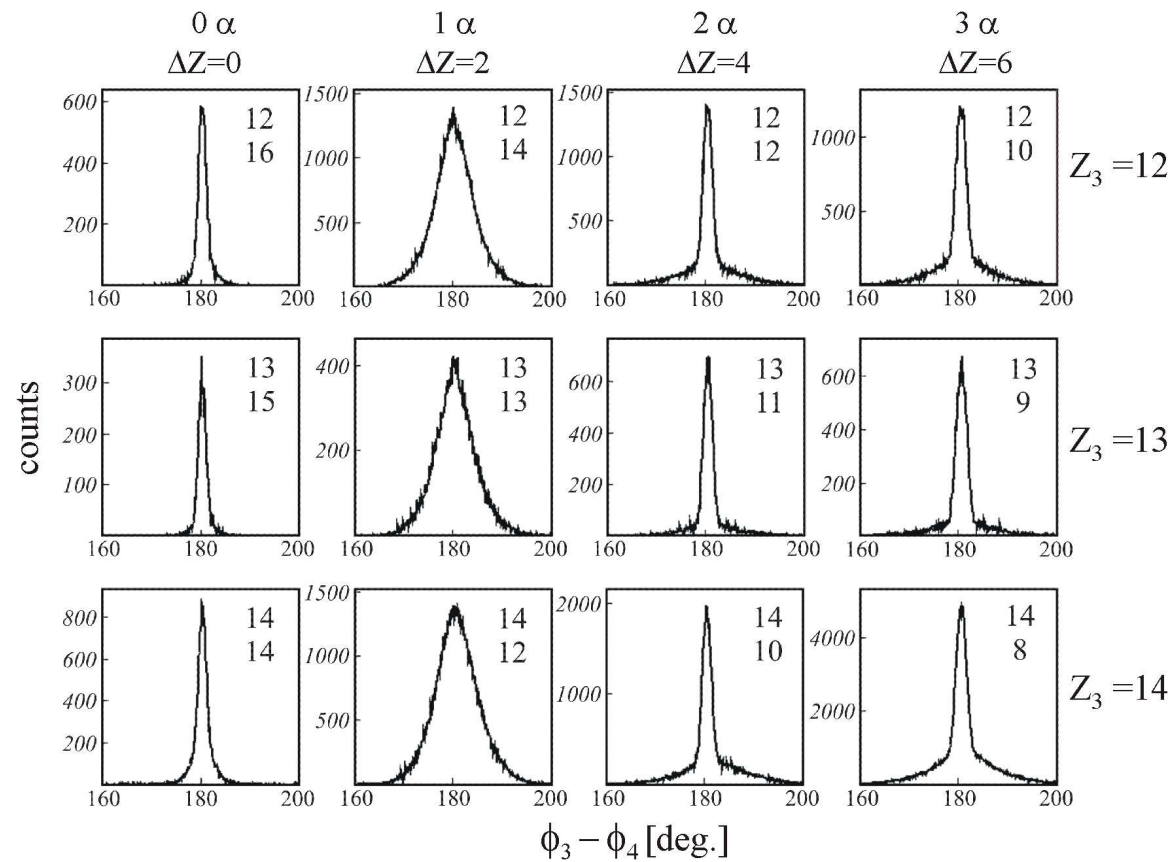

Fig. 5. Yields (counts), $N\left(Z_{3}, Z_{4}\right)$ for $\left(Z_{3}+Z_{4}\right)=$ even, of coincident fragments with charges $Z_{3}$ and $Z_{4}$, as indicated, as a function of the out-of-plane angles $\left(\phi_{3}-\phi_{4}\right)$, in degrees (note the different vertical scales). The out-of-plane angular correlations show differing widths for binary decay (col.1) and for the respective non-binary emission channels with missing $\Delta \mathrm{Z}=1 \alpha(\operatorname{col} .2)$, $2 \alpha(\operatorname{col} .3)$, and $3 \alpha(\operatorname{col} .4)$ in the reaction ${ }^{32} \mathrm{~S}+{ }^{24} \mathrm{Mg}$ at $E_{l a b}=163.5 \mathrm{MeV}$, see also Fig.7.

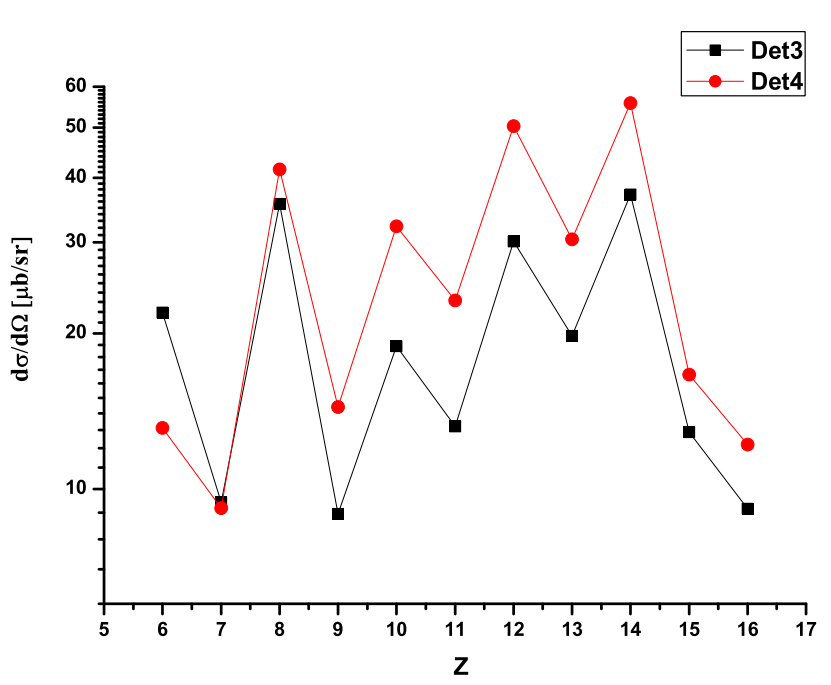

Fig. 4. (Color online) Inclusive coincidence yields, obtained with a gate set for Z-identification in either of the detectors the detectors (3) or (4) for the reaction ${ }^{32} \mathrm{~S}+{ }^{24} \mathrm{Mg} \rightarrow Z_{3}+Z_{4}+\Delta Z$.

events correspond to binary fission with an excitation energy being sufficiently high for one $\alpha$-particle to be evaporated from either of the fission fragments. Note that the top of these distributions are not flat, a very small nar- row component can also be extracted from these spectra (refs. $[12,13])$ these would correspond to ternary coplanar decays with one missing $\alpha$-particle. There are other possible combinations of $\left(Z_{3}, Z_{4}\right)$, which give essentially the same picture, however, with differing yields.

The expectation is that for larger charge losses $\Delta Z>$ 2 (a sequential emission of several charged particles) the $\phi$-correlations will have increasing width (as observed in refs. $[11,12])$. This is fulfilled with a broader component, e.g. for two missing $\alpha$-particles, $\Delta Z=4$ (col.3), and for $\Delta Z=6$ (col.4) in Fig. 5. For $\Delta \mathrm{Z}=4$ and 6 small broad components are observed. For $\Delta \mathrm{Z}=8$ the $\mathrm{Q}$-values are very negative and no contributions from the fission of ${ }^{56} \mathrm{Ni}$ can be expected.

An appreciable contribution from reactions on ${ }^{16} \mathrm{O}$ in the target is present as purely binary events in the narrow part of $\Delta Z=4$, and the corresponding $(-1 \alpha)$-emission channel within the broad part for $\Delta Z=6$. The latter is, however, rather small (see below). The narrow components in $\Delta \mathrm{Z}=4,6$ are stronger (factor 2-3) compared to the yield of the purely binary case, except for the channels with $\left(\mathrm{Z}=8,{ }^{16} \mathrm{O}\right)$. As we will see later, the narrow parts, after subtraction of the ${ }^{16} \mathrm{O}$ contribution can be described (as in ref. [11,13]) by coplanar ternary cluster decay.

Whereas the purely binary decay has a small yield, the yields corresponding to the emission of $1 \alpha$-particle are the largest at this incident energy (see also $[11,13$, $24]$ ), they are broad in the $\phi$-correlations due to the momentum spread induced by the recoil in the evaporation process. The yields in these broad distributions are typ- 

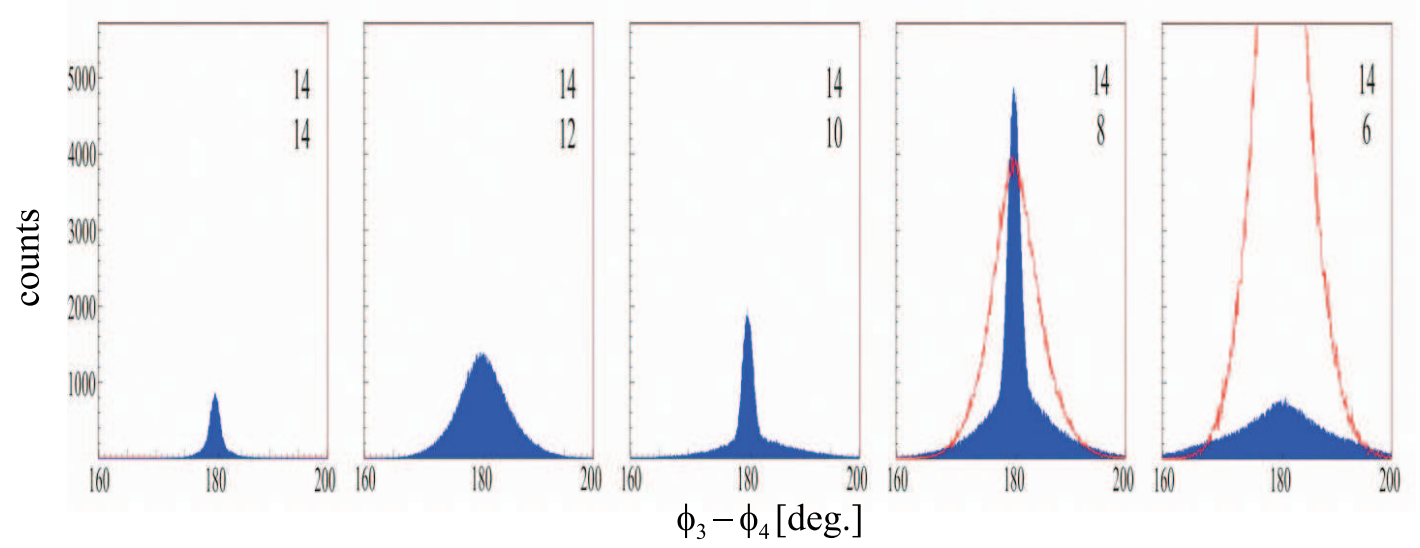

Fig. 6. (Color online) Out-of-plane angular correlations $\mathrm{N}\left(\phi_{3}-\phi_{4}\right)$ with a fixed gate on $Z_{4}$ for binary coincidences (compare with Fig. 5) and the respective emission channels with missing $\Delta \mathrm{Z}=(1-4) \alpha$. The expected broad yield in $\Delta Z=6,8$ are shown for the assumption that the narrow components in $\Delta Z=4,6$ are fully due to the contaminants ${ }^{16} \mathrm{O}$ and ${ }^{12} \mathrm{C}$, respectively.

ically 5-8 times stronger than those of the purely binary channel. This fact gives us the possibility to estimate the contributions from the potential target contaminants, for the reaction on ${ }^{16} \mathrm{O}$ : e.g. ${ }^{32} \mathrm{~S}+{ }^{16} \mathrm{O} \rightarrow{ }^{28} \mathrm{Si}+{ }^{20} \mathrm{Ne}$ (corresponding to $\Delta \mathrm{Z}=4$ from the target ${ }^{24} \mathrm{Mg}$ ). If all events in the narrow components in the $\Delta \mathrm{Z}=4$ would originate from a binary process on ${ }^{16} \mathrm{O}$, a corresponding strong wide component from the reaction ${ }^{32} \mathrm{~S}+{ }^{16} \mathrm{O} \rightarrow{ }^{28} \mathrm{Si}+{ }^{16} \mathrm{O}+1 \alpha$ (about factor 5) must appear in $\Delta \mathrm{Z}=6$ (see Fig. 6 , thin (red) line distribution). For this we assume the centre of mass cross sections for the fission of ${ }^{56} \mathrm{Ni}$ and ${ }^{48} \mathrm{Cr}$ to be approximately the same. This assumption is based of the following arguments: The maximum L-value reached for ${ }^{48} \mathrm{Cr}$ is $10 \%$ smaller giving a $30 \%$ smaller total cross section. However, from the statistical model prediction the number of open channels decreases with decreasing excitation energy in ${ }^{48} \mathrm{Cr}$, giving a larger cross section for the mentioned individuel channels, thus compensating the beforementioned effect. The broad component in $\Delta \mathrm{Z}=6$ is smaller than expected from these considerations, the event rate is a factor 2.5 to small (see also the same procedure for the fission of in ${ }^{60} \mathrm{Zn}$ ref. $\left.[11,13]\right)$. The narrow components from ${ }^{12} \mathrm{C}$ in the target, e.g. reaction ${ }^{32} \mathrm{~S}+{ }^{12} \mathrm{C} \rightarrow$ ${ }^{28} \mathrm{Si}+{ }^{16} \mathrm{O}$ corresponds to $\Delta \mathrm{Z}=6$ relative to the target ${ }^{24} \mathrm{Mg}$. Similarly, We can assume that the observed narrow $\Delta \mathrm{Z}=8$ component originates from ${ }^{12} \mathrm{C}$ alone, however, based on yield in the narrow $\Delta \mathrm{Z}=6$ component the the expected yield of the $(-1 \alpha)$-broad component in $\Delta \mathrm{Z}=8$ should be 4 times larger then observed, actually for purely binary channels from ${ }^{12} \mathrm{C}$, the kinematic conditions for the sum energies and angles are unfavourable in the regions of $\theta_{L}=12.5^{\circ}-45.5^{\circ}$ spanned by the BRS, thus binary reactions on ${ }^{12} \mathrm{C}$ can only give small contributions. With these observations we find that the narrow distributions in the yields $\mathrm{N}\left(\phi_{3}, \phi_{4}\right)$ are only partially due to the reactions on the ${ }^{24} \mathrm{Mg}$ target, however, we are able to subtract the contributions from the contaminants. The quantitative determination of the amount of the contributions from contaminants is discussed below.

For the further discussion of the contaminants we investigate the correlation with odd total charges (see Fig. 7). No very narrow peaks appear in $\Delta \mathrm{Z}=1$, because of the emission of a proton. The increase of the width from $\Delta \mathrm{Z}$ $=1$ to $\Delta \mathrm{Z}=3$ is clearly due to the (additional) emission of the $\alpha$-particle. For $\Delta \mathrm{Z}=5$, however, we observe again a narrower peak as observed in $\Delta \mathrm{Z}=1$. The $\mathrm{Q}$-value for the loss of $2 \alpha$-particles and 1 proton for the ${ }^{24} \mathrm{Mg}$-target is very negative (see Tab. ), so that this contribution to $\Delta \mathrm{Z}=5$ is very small. We can safely assume that the third column in Fig. 7 corresponds completely (almost $100 \%$ ) to the reaction with $\Delta \mathrm{Z}=1$ on the ${ }^{16} \mathrm{O}$ in the target, which has a less negative Q-value. From this assumption, we can determine the ${ }^{16} \mathrm{O}$-content by assuming that the fission into fragments with $(-1 \mathrm{p})$-evaporation on both ${ }^{16} \mathrm{O}$ and on ${ }^{24} \mathrm{Mg}$ have the same cross section (in the cm-system) as done before for the $-1 \alpha$. Again the fission cross section of the related compound nucleus ${ }^{48} \mathrm{Cr}$ at slightly smaller angular momentum will be smaller by $30 \%$, however, due the expected lower excitation energy of the fragments increased cross sections are expected in the $-1 p$ and $-1 \alpha$ channels. With the assumption of the equal $-1 \mathrm{p}$ cross section for the ${ }^{48} \mathrm{Cr}$ and ${ }^{56} \mathrm{Ni}$ cases, we obtain a $45 \%{ }^{16} \mathrm{O}$-content in the ${ }^{24} \mathrm{Mg}$-target of $240 \mu / \mathrm{cm}^{2}$, with an uncertainty up to $60 \%$ The absolute values (after subtraction of the contaminants) of the differential cross sections in the cited channels shown in Fig. 8 are actually very close to those observed in ref. [11,12] for the fission of ${ }^{60} \mathrm{Zn}$ with slightly higher excitation energy and angular momentum. This agreement between two completely independent experiments is very important. A difference appears for the compound nucleus ${ }^{56} \mathrm{Ni}$ with the high yield of $\left(Z_{3}+Z_{4}=14+8\right)$, which is observed in both the broad (this can not be influenced by ${ }^{16} \mathrm{O}$-contributions) and the narrow components. Actually the Q-values may also favour a true ternary decay(see Tab. ) 


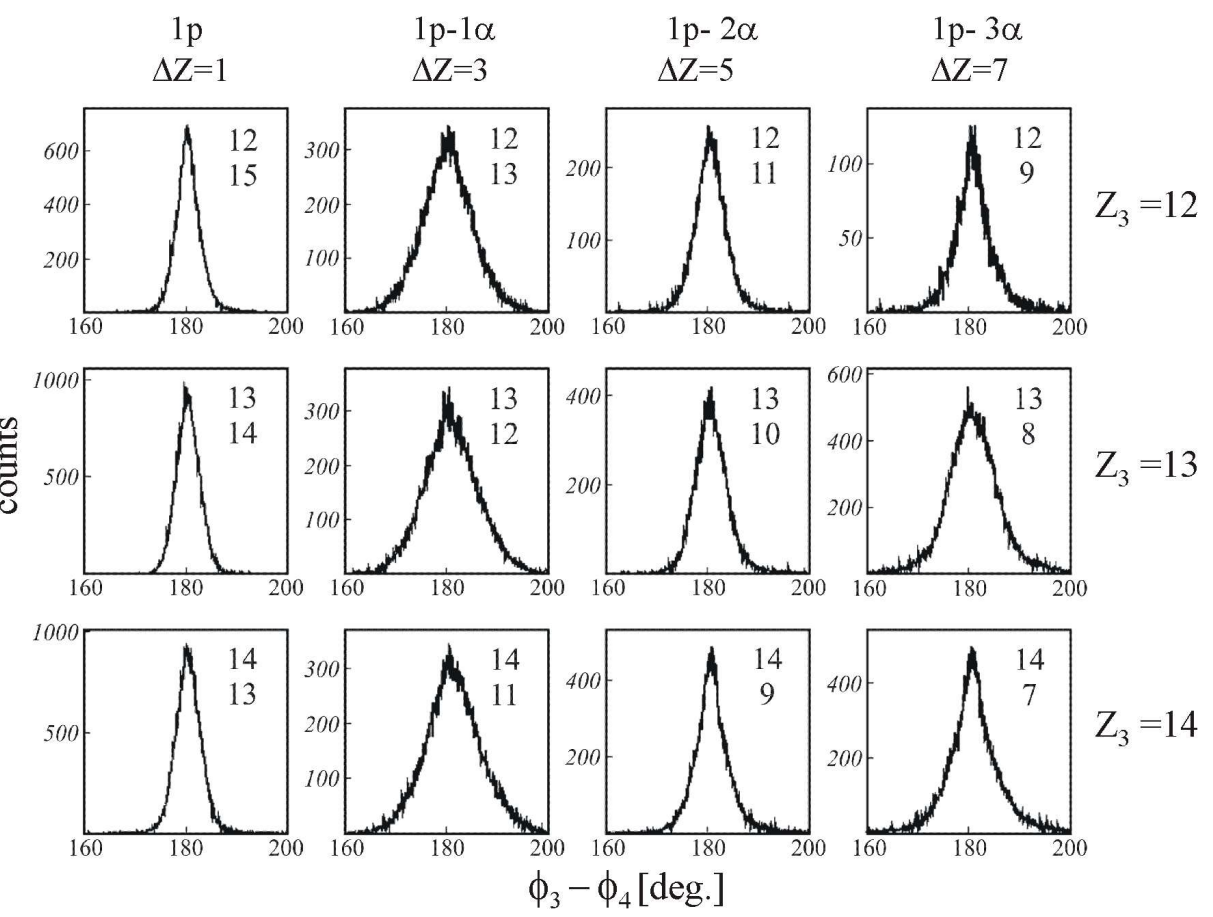

Fig. 7. Yields, $N\left(Z_{3}, Z_{4}\right)$ for $\left(Z_{3}+Z_{4}\right)=$ odd, as a function of $\left(\phi_{3}-\phi_{4}\right)$, in degrees (out-of-plane angular correlations) for coincident fragments with charges as indicated.

\section{Reaction mechanisms}

As an overview on the reaction mechanism giving the narrow components in the $\phi$-correlations, we can consider three different mechanisms.

i) Pre-fission emission of $\alpha$-particles: fission of the $\mathrm{CN}$ after the emission of nucleons or of one or two (and even more) $\alpha$-particles. Such pre-scission process will not disturb the correlation of the two remaining fission fragments. We can rule out this process, because a second chance fission $[17,26]$ after particle emission is very unlikely. From the systematics of Morgenstern [27] the average energy carried by one nucleon is $16.4 \mathrm{MeV}$ and by one $\alpha$-particle is $23.4 \mathrm{MeV}$. In the pre-equilibrium emission of one nucleon or $\alpha$-particle this amount of energy or more must be removed. Furthermore, no significant contribution from a narrow peak in the $\phi$-correlations is observed for the fragment-fragment coincidences with one missing charge or for $\Delta \mathrm{Z}=2$. In the latter case a small narrow component can be extracted, because the distributions do not exhibit a "flat" top.

ii) A strong spin alignment of the binary fission fragments: e.g. for $\Delta \mathrm{Z}=4$ (with sequential emissions) with their angular momenta completely aligned perpendicular to the reaction plane. The alignment from the first step has to be preserved for the whole set of data. The fact that narrow $\phi$-correlations still appear for $\Delta \mathrm{Z}=4,6$ makes it rather unlikely that such a special correlation is created and persists through all decays.

iii) Ternary fission: the missing $\alpha$-particles have only a small momentum perpendicular to the reaction plane. A prompt (simultaneous) ternary decay with the $\alpha$-particles in the neck is unlikely, rather we must consider two subsequent fission processes in a time sequence with two neck ruptures in a short time interval. In this case the emission of the third fragment in the second step would occur in the reaction plane [12].

If the third clustered fragments are preformed in the neck of the composite nucleus the two remaining heavier fragments are emitted in a sharp correlation as in the case of a purely binary fission process. As explained below, due to the energy balance the ternary process can only occur for the highest angular momenta, and the decay then has to be collinear, and the value of $\left(\phi_{3}-\phi_{4}\right)$ remains $180^{\circ}$. We note that in an earlier measurement, performed with the BRS-spectrometer for the reaction ${ }^{36} \mathrm{Ar}+{ }^{24} \mathrm{Mg}(\mathrm{CN}$ $\left.{ }^{60} \mathrm{Zn}\right)$ at $E_{l a b}=195 \mathrm{MeV}$, under similar conditions the same unique narrow correlations with $\left(\phi_{3}-\phi_{4}\right)=180^{\circ}$ have been observed $[11,24]$.

The definition of reaction channels is obtained by the choice of the sum $\left(Z_{3}+Z_{4}\right)$ and the broad or narrow part of the $\phi$-correlations. The corresponding differential cross sections are shown in Fig. 8, they represent the average value for the angular ranges (centre of mass) of ca $70^{\circ}$ $125^{\circ}$. The angular distribution in the mentioned range are flat $[11,12,22]$ and are symmetric around $90^{\circ}$ as in the case of ${ }^{60} \mathrm{Zn}$. For the broad part, interpreted as binary fission with a corresponding excitation in the fragments for particle evaporation to occur, a subtraction of the ${ }^{16} \mathrm{O}$ contribution is made for the $-3 \alpha$-channel. For the narrow component (ternary fission) in the $(-2 \alpha$ and $-3 \alpha)$-channels the reduction in both cases is by a factor 0.45 . The strong 

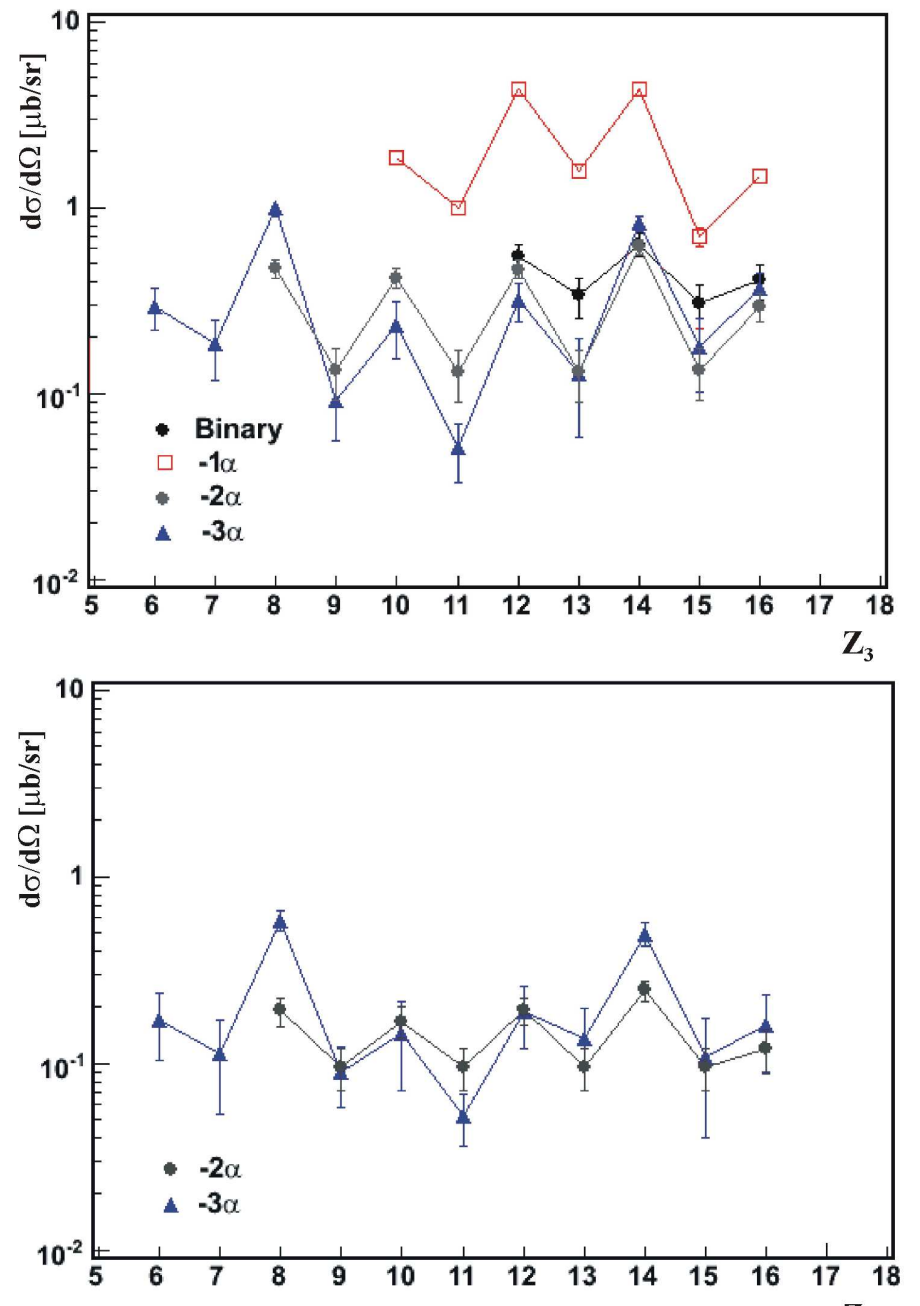

Fig. 8. (Color online) Yields of fission decay of ${ }^{56} \mathrm{Ni}$ : upper part, binary, with $(\Delta Z=0,2,4,6)$ and lower part, ternary cluster decay with missing charges $(\Delta Z=4,6)$. The yields are obtained by selecting one fragment $Z_{3}$ and a coincident fragment. For respective non-binary emission channels the missing charges are $\Delta \mathrm{Z}=(2)-1 \alpha,(4)-2 \alpha$, and (6)-3 $\alpha$-particles .

odd-even effect in the yields is as expected from statisticalmodel predictions, which was also discussed in ref. [11,12].

In the statistical model calculations of [25] the yield for this channel is enhanced due to the favourable Q-value, this work also shows the odd-even effect in the yields. The differences in the yields for the different $\mathrm{Q}$-values between the odd and even channels, and the more favourable Qvalue (by $5 \mathrm{MeV}$ ) of the channels with $\mathrm{Z}=8$ are consistent with the presently observed higher yields. The general behaviour is very similar to the result for ${ }^{60} \mathrm{Zn}$, there no preference of channels with ${ }^{16} \mathrm{O}$ is observed, and not expected from their Q-values.

The higher yield in the channels with $\mathrm{Z}=8$ could also be explained by the preferential decay of a hyper-deformed resonant state, which is known at exactly the chosen excitation energy in the ${ }^{56} \mathrm{Ni}$ nucleus (populated due to the choice of the incident energy on top of ${ }^{28} \mathrm{Si}+{ }^{28} \mathrm{Si}$ resonance), described in $[2,30]$. The shape as predicted in the cluster model is shown in Fig. 9, the formation of ${ }^{16} \mathrm{O}$ is favoured for all shapes.
From the detailed analysis we can also claim that there are again the narrow components in the $\phi$-correlations in binary events, which appear at $\left(\phi_{3}-\phi_{4}\right)=180^{\circ}$. This result point at least to a coplanar ternary cluster decay. For this fact a comment on the geometrical shape of the fission saddle and the three-body configuration is needed. The three fragments could be placed at different relative orientations, however, it can easily be shown that for larger values of the total spin $J$, the collinear configuration, which has the largest moment on inertia relative to all others, gives the lowest fission barrier. This feature has been calculated for some specific cases by Wiebecke and Zhukov [28]. Thus we indeed expect a collinear decay with $\left(\phi_{3}-\phi_{4}\right)=180^{\circ}$, with the ternary fission dominantly occurring at the highest angular momenta. The result also indicates that the missing particles are multiples of $\alpha$-clusters. Such behaviour is predicted by the $\alpha$-cluster model in [2] for the hyper-deformed shapes of ${ }^{56} \mathrm{Ni}$ at high angular momentum, which are shown in Fig. 9. From this figure we may also conclude that the triaxial configuration may dominantly decay into ${ }^{24} \mathrm{Mg}+2 \alpha+{ }^{24} \mathrm{Mg}$, whereas 

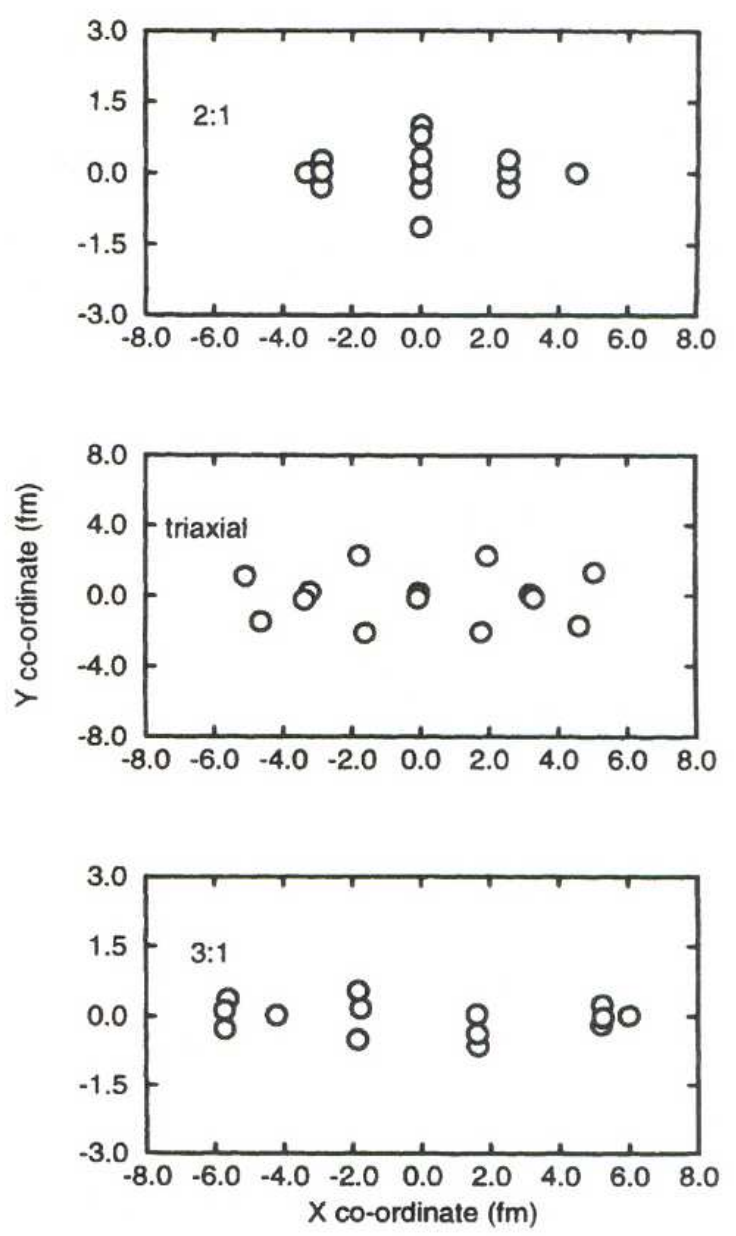

Fig. 9. Clusterisation of the highly deformed ${ }^{56} \mathrm{Ni}$ nucleus in the Brink-Bloch-Cluster model as obtained by Zhang et al. [2] . Different binary and ternary decay channels with the formation of ${ }^{28} \mathrm{Si},{ }^{24} \mathrm{Mg},{ }^{16} \mathrm{O}$ and ${ }^{12} \mathrm{C}$ are expected to be favoured.

the axially symmetric one would decay into ${ }^{16} \mathrm{O}+3 \alpha+$ ${ }^{28} \mathrm{Si}$. Both channels strongly contribute to the ternary fission.

\section{Statistical model for fission}

A major point in the interpretation of the data is to explain the strong yield of the "ternary" fission channels in view of their more negative Q-values. However, e.g. for the binary decay with $3 \alpha$-particles emitted the fragments must be excited, the Q-values are thus similar to the ternary decay with $3 \alpha$-particles missing. For the interpretation of the data as a fission process from an equilibrated CN, we have to consider the statistical phase space for both binary and coplanar ternary fission. This can be achieved by considering the Extended Hauser-Feshbach Method (EHFM) [26]. For ternary events, implying $\mathrm{N} \alpha$ particles in the neck, we make the assumption that their momentum in the centre of mass is small. We will disregard the phase space of these particles, as well as their kinetic energy. For a CN with excitation energy, $E_{C N}^{*}$, a ternary Q-value $Q_{g s}(3,4)$, and excitation energies of the fragments given by $U_{3}, U_{4}$, with their relative kinetic energy as $E_{k i n}(3,4)$, we have the constraint for the excitation energies: $U_{3}+U_{4}=E_{C N}^{*}+Q_{g s}(3,4)-E_{k i n}(3,4)$. The two excitation energies are connected, both fragments being registered in coincidence before further decay. The excitation energies, $U_{3}$ and $U_{4}$, are below the $\alpha$-decay threshold, which for even-even nuclei with $(\mathrm{N}=\mathrm{Z})$ is in the region of 5-8 MeV.

The differential cross section for different mass partitions $(i, j)$ depends on the product of the level densities $\rho_{i}\left(U_{i}, J_{i}\right), \rho_{j}\left(U_{j}, J_{j}\right)$, for $i=3, j=4$ and on the "inverse fusion" cross section $\sigma\left(E_{k i n}(3,4), R_{s}, J\right)$

$\frac{d \sigma(3,4)}{d \Omega d E_{k i n}(3,4)}=C \rho_{3}\left(U_{3}, J_{4}\right) \rho_{4}\left(U_{3}, J_{4}\right) \sigma\left(E_{k i n}(3,4) R_{s}, J\right)$

$J_{3}$ and $J_{4}$ are the spins of the fragments (with total spin $J$ ), and $R_{s}$ distance between fragments for the choice of the configuration $\left(R_{s}=R_{3}+R_{4}+\mathrm{d}\right.$, where $\mathrm{d}$ is a neck parameter). For the total energy balance, with potential energy $V_{\text {pot }}^{\text {eff }}\left(J, 3,4, R_{s}\right)$, which includes the rotational energy. We define the free energy which is available for the fragments to be excited (excitation included in the definition of $Q(3,4))$ or emitted in their ground states:

$$
E_{f r e e}(3,4, J)=E_{C N}^{*}+Q(3,4)+V_{p o t}^{e f f}\left(J, 3,4, R_{s}\right)
$$

This value will determine the yield for a particular partition. The rotational energy $E_{\text {rot }}\left(J, 3,4, R_{s}\right)$ depends on the total spin $J$ and on the moment of inertia $\Theta_{f f}\left(R_{s}\right)$. The total potential contains the shell corrections $\Delta_{s h}\left(R_{s}\right)$ at the deformed saddle point: $V_{\text {pot }}^{\text {eff }}\left(J, 3,4, R_{s}\right)=$ $E_{\text {rot }}\left(J, 3,4, R_{s}\right)+V_{\text {pot }}\left(3,4, R_{s}\right)+\Delta_{s h}\left(R_{s}\right)$. The values of the shell corrections (in the range of $5-8 \mathrm{MeV}$ ) to the saddle point of hyper-deformed shapes are particular large for $\mathrm{N}=\mathrm{Z}=28$, and can be found in the review of Ragnarson et al. [6].

The interpretation of the relative yields of the binary and ternary fission yields can be obtained from these considerations, they will be determined by:

a) the Q-values, namely different values of $E_{\text {free }}(3,4, J)$, also different values of $U_{i}$ and $U_{j}$ needed for particle evaporation,

b) the shell corrections for large deformations (3:1 axis ratio),

c) the angular momentum. Because of the different moments of inertia, $\Theta_{f f}$, for binary (normally deformed) and ternary (hyper-deformed) shapes, the corresponding differences in fission barrier heights become smaller at high $J$ values (see Fig. 10). Some relevant values are summarised in Table 1. We also point to the dependence of the yield on the mass asymmetry and angular momentum as discussed e.g. in ref.[17]: at low angular momentum asymmetric mass splits are favoured, whereas at for large values of $J$ a flat distribution as in Fig. 8 as function for $Z_{3,4}$ is predicted. 


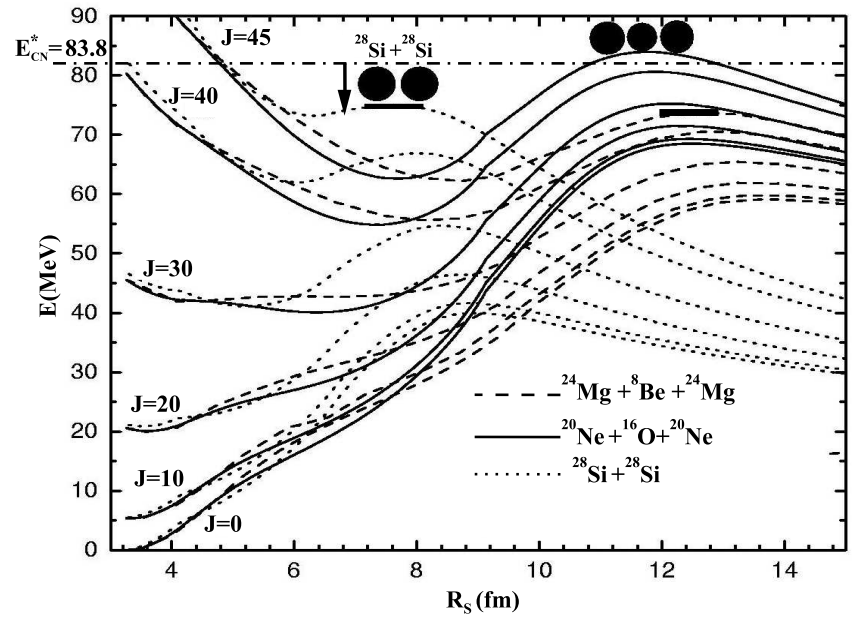

Fig. 10. Potential energies for selected fragmentations in the decay of ${ }^{56} \mathrm{Ni}$ as a function of the deformation (represented by the distance $R_{z}$ between the two heavier fragments) for different angular momenta (in units of $\hbar$ ) for binary and ternary fission, respectively. Channels with different $\Delta \mathrm{Z}$, (missing 2 and $4 \alpha$-particles) are shown. The barriers and the free energy (indicated by the arrow) are marked. The Q-value for, ${ }^{28} \mathrm{Si}+{ }^{28} \mathrm{Si}$ is $+3.04 \mathrm{MeV}$, and $\mathrm{Q}=-16.9 \mathrm{MeV}$ for the ternary decay with ${ }^{8} \mathrm{Be}(-2 \alpha)$.

Table 1. Q-values (Q), the inverse of the moments of inertia $\left(\hbar^{2} / 2 \Theta_{f f}\right)$ and barrier heights $\mathrm{B}_{f f}$ for $\mathrm{J}=45 \hbar$ (in $\mathrm{MeV}$ ) for some fission channels of ${ }^{56} \mathrm{Ni}$

\begin{tabular}{|l|l|l|l|}
\hline Reactions & $\hbar^{2} / 2 \Theta_{f f}$ & $B_{f f}$ & $\mathrm{Q}[\mathrm{MeV}]$ \\
\hline Bin, $-0 \alpha,{ }^{28} \mathrm{Si}+{ }^{28} \mathrm{Si}$ & 0.038 & 74.8 & +3.04 \\
Bin, $-0 \alpha,{ }^{20} \mathrm{Ne}+{ }^{36} \mathrm{Ar}$ & 0.041 & 81.3 & -2.67 \\
Bin, $-0 \alpha,{ }^{22} \mathrm{Na}+{ }^{34} \mathrm{Cl}$ & 0.040 & 88.6 & -10.3 \\
Bin, $-1 \alpha,{ }^{24} \mathrm{Mg}+{ }^{28} \mathrm{Si}$ & & & -6.947 \\
Bin, $-1 \alpha,{ }^{16} \mathrm{O}+{ }^{36} \mathrm{Ar}$ & & & -7.405 \\
Bin, $-1 \alpha,{ }^{22} \mathrm{Na}+{ }^{30} \mathrm{P}$ & & & -16.991 \\
Bin, $-1 \alpha,{ }^{26} \mathrm{Al}+{ }^{26} \mathrm{Al}$ & & & -17.953 \\
\hline Tern, $-2 \alpha,{ }^{24} \mathrm{Mg}+{ }^{24} \mathrm{Mg}$ & 0.015 & 73.7 & -16.93 \\
Tern, $-2 \alpha,{ }^{26} \mathrm{Al}+{ }^{22} \mathrm{Na}$ & & & -27.40 \\
Tern, $-2 \alpha,{ }^{28} \mathrm{Si}+{ }^{20} \mathrm{Ne}$ & & & -16.26 \\
\hline Tern, $-3 \alpha,{ }^{24} \mathrm{Mg}+{ }^{20} \mathrm{Ne}$ & 0.014 & 78.9 & -26.24 \\
Tern, $-3 \alpha,{ }^{22} \mathrm{Na}+{ }^{22} \mathrm{Na}$ & 0.0154 & 89.5 & -36.86 \\
Tern, $-3 \alpha,{ }^{28} \mathrm{Si}+{ }^{16} \mathrm{O}$ & & & -20.99 \\
Tern, ${ }^{28} \mathrm{Si}+{ }^{12} \mathrm{C}+{ }^{16} \mathrm{O}$ & & & -13.34 \\
$-1 p, 2 \alpha,\left({ }^{24} \mathrm{Mg}\right),{ }^{20} \mathrm{Ne}+{ }^{27} \mathrm{Al}$ & & & -27.85 \\
\hline \hline Bin (on $\left.{ }^{16} \mathrm{O}\right),{ }^{24} \mathrm{Mg}+{ }^{24} \mathrm{Mg}$ & & & -2.88 \\
$-1 p,\left(\right.$ on $\left.{ }^{16} \mathrm{O}\right),{ }^{16} \mathrm{O}+{ }^{31} \mathrm{P}$ & & & -8.86 \\
$-1 \alpha,\left(\right.$ on $\left.{ }^{16} \mathrm{O}\right),{ }^{16} \mathrm{O}+{ }^{28} \mathrm{Si}$ & & & -6.95 \\
\hline
\end{tabular}

In the calculations by Royer [9], the liquid-drop energies, the Q-values and the rotational energies constitute the main part of the barrier heights for the fission process. With the angular momentum dependence of $V_{\text {pot }}^{e f f}\left(J, 3,4, R_{s}\right)$ the free energy $E_{\text {free }}(3,4, J)$ at the saddle point is dramatically reduced (see Fig. 10) for both the binary and the ternary mass splits as a function of $J$. The ternary barrier becomes comparable to the binary barrier, and the difference in Q-values being compensated by the smaller value of $E_{\text {rot }}\left(J, 3,4, R_{s}\right)$. In Table 1 we show the Q-values and the rotational energies at the saddle point for $J=45 \hbar$. The Q-values are more negative for ternary mass splits, therefore negligible contributions are expected at low angular momentum. The ternary fission process from the hyper-deformed configuration is expected to be enhanced due to a lowering of its ternary fission barrier by the shell corrections. The Q-values for the ternary mass splits with odd-odd charge fragments are in addition 5-10 MeV more negative. Thus, in these cases much lower yields and less subsequent decays via particle evaporation are possible.

\section{Conclusion}

We conclude that the observation of the narrow coplanar fission fragment coincidences in the present data, in conjunction with the earlier work on the same phenomenon for ${ }^{60} \mathrm{Zn}$ in [11-13], is a unique feature, which can only be observed with the experimental set-up presented. It gives evidence for the occurrence of ternary cluster decay processes in excited $\mathrm{N}=\mathrm{Z}$ nuclei, the two cases ${ }^{60} \mathrm{Zn},{ }^{56} \mathrm{Ni}$ have been studied at similar excitation energy $(88 \mathrm{MeV}$ and $83.5 \mathrm{MeV})$ and angular momentum $(42-45 \hbar)$. The two rather independent experiments gave absolute (and relative) cross sections for binary and ternary fission decays which are comparable (within factors of 1.5-2).

The ternary coplanar fission is a signature of the decay from an extremely deformed nucleus at high angular momentum. Although a detailed analysis within the statistical model still needs to be undertaken, the relative yields in the different channels clearly show the odd-even staggering expected as a function of charge from the statistical model. with the lower yields for the negative Q-values in the odd-odd channels. Remarkable is the preference for particular cluster decays, also expected from considerations of the the statistical phase space. The $\mathrm{Q}$-values with ${ }^{16} \mathrm{O}$ in the final channel are always typically higher by 4$5 \mathrm{MeV}$ and the channel ${ }^{28} \mathrm{Si}+{ }^{12} \mathrm{C}+{ }^{16} \mathrm{O}$ can be favoured in addition. The result indicates that the highly deformed nucleus ${ }^{56} \mathrm{Ni}$ is clustered, and the formation of a resonance (obtained by the choice of the incident energy) with a particular shape may contribute to the observed fission yield systematics. The populated state is well described in the Brink-Bloch cranked $\alpha$-cluster model [2], its shape was shown in Fig. 9.

This work also shows that the search for structures with hyper-deformation in rapidly rotating nuclei, can be pursued with charged-particle spectroscopy. The neck represents a region of low nuclear density favouring the formation of $\alpha$-clusters as discussed by Horiuchi [29]. For nuclei in the medium-mass region a complete reconstruc),tion of the ternary fission events can be undertaken with appropriate detector systems. Thus, measurements of the ternary fission process offer the possibility of a detailed spectroscopy of extremely deformed nuclear states. 


\section{Acknowledgements}

We thank the VIVITRON crew for their excellent support. We would like to acknowledge the help of the EUROBALL group of IReS during the experiment. This work was supported by the ministry of research (BMBF, Germany) under contract Nr.06-OB-900, and by EC-Euroviv contract HPRI-CT-1999-0078. Tz. Kokalova and V. Zherebchevsky thank the DAAD for their support. We thank C. Wheldon for his numerous contributions in this project.

\section{References}

1. S. Cohen, F. Plasil and W. J. Swiatecki, Ann. Phys. (N.Y.) 82 (1974) 557.

2. J. Zhang, A. C. Merchant, and W. D. M. Rae, Phys. Rev. C 49 (1994) 562 and W.D.M. Rae in Proc., $5^{\text {th }}$ Intern. Conf. on Clustering Aspects in Nuclear and Subnuclear Systems 1988, Kyoto, Prog. Theor. Phys. (Jap.) ed. K. Ikeda, (1989) p. 80.

3. G. Leander and S. E. Larsson, Nucl. Phys. A 239 (1975) 93.

4. S.Åberg, H. Flocard and W. Nazarewicz, Ann. Rev. Nucl. Science, Vol. 40 (1990) 439.

5. S. Aberg and L.O. Joensson, Z. Phys. A 349 (1994) 205.

6. I. Ragnarsson, S. Aberg and R. K. Sheline, Phys. Scr. 24, 215 (1981); I. Ragnarsson, S. G. Nilsson and R. K. Sheline, Phys. Rep. 45 (1978) 1.

7. E. Ideguchi, D. G. Sarantites, W. Reviol, A. V. Afanasjev, M. Devlin, C. Baktash, R. V. F. Janssens, D. Rudolph, A. Axelsson, M. P. Carpenter, A. Galindo-Uribarri, D. R. LaFosse, T. Lauritsen, F. Lerma, C. J. Lister, P. Reiter, D. Seweryniak, M. Weiszflog, J. N. Wilson, Phys. Rev. Lett.87, (2001) 222501.

8. G. Royer and F. Haddad, J. Phys. G 21 (1995) 339.

9. G. Royer, J. Phys. G 21 (1995) 249; also G. Royer, F. Haddad and J. Mignen, J. Phys. G 18 (1992) 2015.

10. A. Algora, J. Cseh et al. Phys. Lett. B 639 (2006) 451, and private communication.

11. V. I. Zherebchevsky, W. von Oertzen, et al., Phys. Lett. B 646 (2007) 12.

12. V. I. Zherebchevsky, W. von Oertzen, D. V. Kamanin, JETP Lett. Vol. 85, N.3, (2007) 136. Also Dr.Thesis, St. Petersburg University, 2007.

13. W. von Oertzen et al., submitted to Phys.Rev.C

14. C. Beck and A. Szanto de Toledo, Phys. Rev. C 53 (1996) 1989.

15. B. Herskind et al., 2007, in Acta Phys. Pol. B 38 (2007 14121

16. R. Betts et al., Phys. Rev. Letters 47 (1981) 23.

17. S.J. Sanders, A. Szanto de Toledo and C. Beck, Phys. Rep. 311 (1999) 487 and references therein.

18. B. Gebauer et al., in Proc. Int. Conf. on the Future of Nucl. Spectroscopy, Crete, Greece, 1993, eds. W. Gelletly et al., p. 168.

19. B. Gebauer et al., Achievements with the Euroball spectrometer, 2003, eds. W. Korten and S. Lunardi, p. 135.

20. C. Beck et al., Nucl. Phys. A 734 (2004) 453.

21. C. Wheldon et al., submitted to Nucl.Phys.A

22. G. Efimov Dr.Thesis, Dubna University. 2008.

23. N. Curtis et al., Phys. Rev. C 53 (1996) 1963
24. S. Thummerer et al., Nuovo Cimento 111 A (1998) 1077.

25. A. V. Andreev et al., Eur. Phys. J. A 30 (2006) 579.

26. T. Matsuse, C. Beck, R. Nouicer and D. Mahboub, Phys. Rev. C 55 (1997) 1380.

27. H. Morgenstern et al., Z. Phys. A 313 (1983) 39.

28. H. J. Wiebecke and M. Zhukov, Nucl. Phys. Nucl. Phys. A 351 (1981) 321.

29. H. Horiuchi, Nucl. Phys. A 731 (2004) 329.

30. C. Beck et al., Phys. Rev. C 63 (2001) 014607. 